\section{OPEN ACCESS}

Edited by:

Chad A. Bousman,

University of Calgary, Canada

Reviewed by:

Min Ling,

University of Oklahoma,

United States

Daniel B. Campbell,

Michigan State University,

United States

*Correspondence:

Richard E. Frye

rfrye@phoenixchildrens.com

Specialty section:

This article was submitted to

Molecular Psychiatry,

a section of the journal

Frontiers in Psychiatry

Received: 18 December 2018

Accepted: 31 July 2019

Published: 11 September 2019

Citation:

Harville T, Rhodes-Clark B,

Bennuri SC, Delhey L, Slattery J,

Tippett M, Wynne R, Rose S,

Kahler S and Frye RE (2019)

Inheritance of HLA-CW7 Associated With Autism Spectrum Disorder (ASD).

Front. Psychiatry 10:612.

doi: 10.3389/fpsyt.2019.00612

\title{
Inheritance of HLA-Cw7 Associated With Autism Spectrum Disorder (ASD)
}

\begin{abstract}
Terry Harville 1,2, Bobbie Rhodes-Clark ${ }^{1}$, Sirish C. Bennuri ${ }^{3,5}$, Leanna Delhey ${ }^{4,5}$, John Slattery ${ }^{6}$, Marie Tippett ${ }^{3,5}$, Rebecca Wynne ${ }^{7}$, Shannon Rose ${ }^{3,5}$, Stephen Kahler ${ }^{3,5}$ and Richard E. Frye ${ }^{8,9 *}$
\end{abstract}

\begin{abstract}
${ }^{1}$ Department of Pathology, University of Arkansas for Medical Sciences, Little Rock, AR, United States, ${ }^{2}$ Department of Internal Medicine, University of Arkansas for Medical Sciences, Little Rock, AR, United States, ${ }^{3}$ Department of Pediatrics, College of Medicine, University of Arkansas for Medical Sciences, Little Rock, AR, United States, ${ }^{4}$ School of Public Health, University of Arkansas for Medical Sciences, Little Rock, AR, United States, ${ }^{5}$ Arkansas Children's Research Institute, Little Rock, AR, United States, ${ }^{6}$ BioRosa Technologies Inc, San Francisco, CA, United States, ${ }^{7}$ National Center for Toxicological Research, Jefferson, AR, United States, ${ }^{8}$ Barrow Neurological Institute at Phoenix Children's Hospital, Phoenix, AZ, United States, ${ }^{9}$ Department of Child Health, University of Arizona College of Medicine, Phoenix, AZ, United States
\end{abstract}

Autism spectrum disorder (ASD) is a behaviorally defined disorder that is now thought to affect approximately 1 in 69 children in the United States. In most cases, the etiology is unknown, but several studies point to the interaction of genetic predisposition with environmental factors. The immune system is thought to have a causative role in ASD, and specific studies have implicated $T$ lymphocytes, monocytes, natural killer (NK) cells, and certain cytokines. The human leukocyte antigen (HLA) system is involved in the underlying process for shaping an individual's immune system, and specific HLA alleles are associated with specific diseases as risk factors. In this study, we determine whether a specific HLA allele was associated with ASD in a large cohort of patients with ASD. Identifying such an association could help in the identification of immune system components which may have a causative role in specific cohorts of patients with ASD who share similar specific clinical features. Specimens from 143 patients with ASD were analyzed with respect to race and ethnicity. Overall, HLA-Cw7 was present in a much greater frequency than expected in individuals with ASD as compared to the general population. Further, the cohort of patients who express HLA-Cw7 shares specific immune system/inflammatory clinical features including being more likely to have allergies, food intolerances, and chronic sinusitis as compared to those with ASD who did not express HLA-Cw7. HLA-Cw7 has a role in stimulating NK cells. Thus, this finding may indicate that chronic over-activation of NK cells may have a role in the manifestation of ASD in a cohort of patients with increased immune system/inflammatory features.

Keywords: autism (ASD), HLA, immune system, natural killer cells, innate immnuity 


\section{INTRODUCTION}

Autism spectrum disorder (ASD) is a behaviorally defined disorder that affects approximately 1 in 69 children in the United States (1). The definition of ASD is outlined in the Diagnostic and Statistical Manual of Mental Disorders (DSM)-5 which requires that those diagnosed exhibit deficits in social communication along with the presence of restricted interests and/or repetitive behaviors and/or sensory disorders. In addition, the symptoms must significantly interfere with the individual's ability to function properly in school, work, and other areas of life (2). Despite decades of research, the etiology(ies) of ASD is(are) still unknown (3), but epidemiological studies suggest that genetic predisposition interacts with environmental influences to trigger the biological events that results in ASD (4). Several physiological systems that are known to be influenced by environmental factors have been implicated in ASD, including redox and mitochondrial metabolism, as well as, immune dysregulation $(3,4)$.

ASD is described as a "spectrum" due to the variability in the extent and severity of behavioral and medical symptoms. The diagnostic criteria for ASD is wide enough to capture an overall phenotype, but the variability in symptoms and severity, as well as, specific laboratory results indicates that multiple etiologies may be responsible, including one that involves immune dysfunction (5). One of the challenges is to define potential subtypes using behavioral and physiological biomarkers in order to better define the disorder and identify treatment targets.

Many studies have indicated involvement of the immune system in ASD. Early studies identified family histories of autoimmune disease, specific HLA associations, and autoantibodies to a wide range of brain and non-brain tissues in children with ASD (6-13). Other studies have demonstrated a reduction in immunoglobulin production (14) and a positive therapeutic response to intravenous immunoglobulin treatment (15). Perhaps the most pivotal study from the Kennedy Krieger Institute found that autopsy revealed inflammatory changes in the cerebellum with cytokine elevations in cortical areas and similar abnormal cytokine profiles in the cerebrospinal fluid of patients with ASD (16). However, the reason for this immune dysregulation remains unknown.

Most notably, T lymphocytes and monocytes have been implicated as immune system components involved with ASD. Another immune cell type, natural killer (NK) cells are also implicated (11, 13, 17-19). The human leukocyte antigen (HLA) system is the core of the development and maintenance of the immune system in humans. It is the most polymorphic of all human proteins, and specific polymorphisms have been demonstrated to have specific roles in the development of autoimmune disorders. For example, it is well known that the risk for developing ankylosing spondylitis is increased in individuals who have inherited HLAB27. Thus, involvement of specific HLA components in the etiology of immune-associated ASD is plausible.

NK cells are thought to be most critical as part of the immune system's first-line defense and immunosurveillance. Essentially, all the cells of the body (except for RBC) express HLA class I proteins. The expressed HLA-C locus components interact with NK cell killer immunoglobulin-like receptor (KIR) ligands. The HLA-C locus proteins are divided into two groups, group I or group II, based on the expression of 77 Ser and 80 Asn, in the sequence of the former, and expression 77 Asn and 80 Lys in the sequence of the latter. Further, HLA-C group I and group II reciprocally act as inhibitors and activators with specific KIR ligands expressed on the NK cells.

During normal immunosurveillance, an NK cell interrogating a human cell. If it finds the correct level of expression of HLA-C group I and group II components that "balance" activation and inhibition signaling via its KIR ligands, it leaves the cell unaffected. The normal ratio of expression of HLA-C group I to group II is 0.86 . The presumption is that this was decided via evolution to provide the optimal NK cell immunosurveillance. Significant deviations from this may result in delayed immune system activation or perhaps over activation of immunity. For example, if the cell was infected by a virus, or was transformed and becoming cancerous, then the "normal" levels of HLA-C expression on the cell's surface may be altered, and the NK cell may become activated via the KIR ligand interaction. This activation could generate inflammatory signals, and the NK cell may actively begin to kill the cell.

We hypothesized that a significant involvement of the immune system in ASD would be exhibited as bias in the distribution of specific HLA types. Further, we hypothesized that this would be occurring in a cohort of patients with similar clinical and laboratory features, rather than the entire population of ASD. To this end, we performed HLA typing on 126 patients with ASD and 17 lymphoblastoid cell lines (LCLs) derived from patients with ASD (143 total). We analyzed the typing results based on the normal expected frequencies for total and subpopulations of African-American, Asian, Caucasian, and Hispanic racial and ethnic distributions. We found that HLA-Cw7 was overrepresented in patients with ASD, primarily in the Caucasian cohort. Further analyses for clinical and laboratory features indicate that those who express HLA-Cw7 have a higher rate of immune system-associated issues in comparison with those with ASD who do not express HLA-Cw7. Our data support the notion that the increased expression of HLA-Cw7 in a cohort of patients with ASD and immune system-associated issues may support chronic over-stimulation of NK cells, resulting in the phenotypic features of ASD.

\section{METHODS}

The study contains data derived from cell lines obtained from 126 participants diagnosed with ASD seen in at Arkansas Children's Research Institute (Little Rock, AR) as part of two clinical studies (NCT02000284, NCT01602016) as well as 17 LCLs from the Autism Genetic Resource Exchange (AGRE) and the National Institutes of Mental Health biorepository. The primary purpose of the clinical studies from which these samples were derived was to investigate the relationship between mitochondrial dysfunction and ASD (NCT02000284) and abnormalities in folate metabolism and ASD (NCT01602016). Results on the primary studies from which these samples were derived has been published previously 
(20-28), but investigation of HLA types in relation to ASD has not been previously reported in these samples. Likewise, the LCLs used in this study have been used in previous laboratory studies examining mitochondrial dysfunction in relation to ASD (29-32), and the effect of environmental agents on mitochondrial (33-36) and immune (37) functions of the LCLs but investigation of the HLA types of these LCLs in relation to ASD has not been published previously. The Institutional Review Board (IRB) at the University of Arkansas for Medical Sciences (Little Rock, AR) approved the clinical studies and use of cell lines for the primary investigation and additional investigations such as HLA typing and other secondary genomic, metabolomic, and proteomic investigations. For clinical studies, parents of participants provided written informed consent. All experiments were performed in accordance with relevant guidelines and regulations.

\section{ASD Participants}

One hundred twenty-six individuals with ASD met inclusion and exclusion criteria. Table 1 outlines the clinical characteristics of these participants. Comorbid conditions were both derived from a parent reported medical questionnaire and from review of conditions diagnosed in the medical records. Regression (defined as loss of already obtained skills) was defined in detail in our questionnaire. Questions regarding regression included the timing, specific skills lost, duration of the regression, trigger, and whether multiple regressions were identified. Since this paper is not specifically on regression, we have not summarized these details in the table, only whether regression(s) were present or not present. This method for assessing medical comorbidities has been used in several of our previous studies $(20,21,24,25)$. We then selected the medical conditions that affected $10 \%$ or more of

TABLE 1 | ASD participant characteristics $(N=126)$.

\section{Variable}

Age, mean (SD), years and months

Males, N (\%)

Caucasian

African American

Hispanic

Asian

Comorbid conditions (parent report), \% (N)

Abdominal pain

Allergies

Anxiety

ADHD

Sinusitis

Immune problems

Headaches

Regression

Comorbid conditions (medical records),

\section{N (\%)}

Food allergies/intolerances

Chronic constipation

Chronic persistent infections

Immune disorder

Epilepsy
7 years and 8 months (3 years and 6 months) $82 \%(22 / 126)$ $83 \%(104 / 126)$ $7 \%(9 / 126)$ $3 \%(4 / 126)$ $7 \%(9 / 126)$

$19 \%(23 / 118)$ $43 \%(51 / 118)$ 26\% (30/117) $32 \%(37 / 117)$ $13 \%(15 / 118)$

$14 \%(17 / 118)$

$21 \%(25 / 118)$

$62 \%(72 / 116)$ the individuals in the sample. Conditions with sample prevalence of less than $10 \%$ were considered unlikely to differentiate any immune subgroups defined by the HLA-typing analysis. Data for all the comorbid conditions were not available for all participants, as some families did not complete the questionnaire or did not provide medical records for review, or the review of the medical records could not determine if the participant suffered from the comorbid condition or not. This information was missing at random and affected less than $10 \%$ of the participants. The total number of participants with the required information is outlined in the table below.

Inclusion criteria were (i) age 3 to 14 years of age and (ii) ASD diagnosis. Exclusion criteria were (i) chronic treatment with medications that would detrimentally affect mitochondrial function for the mitochondrial study from which these samples were derived, such as, antipsychotic medications, (ii) vitamin or mineral supplementation exceeding the recommended daily allowance, and (iii) prematurity.

The ASD diagnosis was defined by one of the following: (i) a gold-standard diagnostic instrument such as the Autism Diagnostic Observation Schedule (ADOS) and/or Autism Diagnostic Interview-Revised (ADI-R); (ii) the state of Arkansas diagnostic standard, defined as agreement of a physician, psychologist, and speech therapist; and/or (iii) DSM diagnosis by a physician along with standardized validated questionnaires and diagnosis confirmation by the Principal Investigator at the time (REF). We have validated that this criteria captures an accurate diagnosis of ASD in our previous studies by re-evaluating a portion of the participants with the ADI-R and determining that they all were well within the diagnostic criteria for $\operatorname{ASD}(20,21,24,25)$.

\section{Cell Lines}

As outlined in Supplemental Table 1, 17 LCLs were derived from white males diagnosed with autistic disorder with a pedigree having at least one other affected male sibling (i.e., multiplex family) (mean [SD] age 12.5 [3.0] years). These LCLs were obtained from the Autism Genetic Resource Exchange (AGRE; Los Angeles, CA, USA) and the National Institutes of Mental Health (NIMH; Bethesda, MD, USA) center for collaborative genomic studies on mental disorders. All relevant guidelines and regulations were followed. These de-identified human samples were determined to be exempt from IRB review. Boys from which these autistic disorder LCLs were derived were diagnosed with a gold-standard examination, either the ADOS or the ADI-R.

\section{DNA Isolation}

DNA was isolated from buffy coats of blood collected in EDTA tubes using the Purgene DNA Purification Kit (Gentra Systems, Inc., Minneapolis, MN). For cell lines, DNA was isolated from $5 \times 10^{6}$ pelleted cells using the same kit.

\section{HLA Typing}

HLA typing was performed as previously reported using reagents and supplies from One Lambda ${ }^{\circledR}$, in an American Society of Histocompatibility and Immunogenetics (ASHI) 
certified, and College of American Pathology (CAP) certified, laboratory using the LabType ${ }^{\circledR}$ rSSO technique (38).

\section{Statistical Analyses}

HLA-typing results for the patients and LCLs with ASD were compared with the distributions of HLA components reported by the United Network for Organ Sharing (UNOs) (https:// unos.org/wp-content/uploads/unos/CPRA_frequencies.xls). These data are felt to reflect accurately the distributions of HLA types by race/ethnicity in the US. The racial/ethnic distribution in the specimens with ASD differs from those of the general population (Supplemental Table 2). Therefore, the expected frequency calculations were adjusted to accommodate the deviations by race/ethnicity from the general population. From 143 study subjects, 286 HLA alleles are present. The expected allele frequencies of the general population were determined by taking the fraction of each individual allele present and multiplying by 286 , the total expected allele number. Thus, potential fractions of expected alleles are generated, and these are rounded to the nearest integer. Not all HLA types are found in the patients with ASD in our study. Thus, these cannot be analyzed, but the lack of these HLA types has potential to affect the expected values, since all HLA alleles at the serologic equivalence level were included in making the expected calculations. Therefore, the total numbers of expected alleles for each HLA-locus are projected not to completely add up to 286. Some HLA alleles are null alleles, and therefore not expressed. There were 2 HLA-C locus null alleles and 45 HLA-DR51/52/53 null alleles in the patients with ASD. Therefore, the total number of alleles in patients with ASD for HLA-C and HLA-DR51/52/53 were 284 and 241, respectively. HLA-DR51/52/53 were not analyzed separately by race ethnicity.

Chi-square analyses were performed for samples with larger numbers, and Fisher Exact Test analyses were performed with sample size approximately 20 or fewer. The p values were calculated using Microsoft Excel ${ }^{\circledR}$, with $\mathrm{p}<0.001$ selected to represent the most stringent condition for determining an association with specific HLA types. Results were analyzed similar to previous studies searching for associations of HLA-typing results with disease $(6-12,13,39)$.

To determine if the specific HLA subgroup identified was defined by specific medical symptoms, partial least squares discriminant analysis (PLSDA) using $\mathrm{R}$ version 3.5.0 mixOmics Omics Data Integration Project (version 6.3.2) was used to explore symptoms depicted in Table 1 to determine if there were any specific symptoms that differentiated subgroups identified by the HLA-typing results.

\section{RESULTS}

\section{HLA Typing}

HLA typing was performed on 143 total subjects (patients with ASD and LCLs from those diagnosed with ASD). The results were compared with the frequencies of individual HLA components from a very large national database (https://unos.org/wp-content/ uploads/unos/CPRA_frequencies.xls), adjusted for the race/ ethnic distributions of the subjects with ASD. The total group of patients was analyzed (see Supplementary Tables 3-8), and the HLA types were separately analyzed based on the individual race/ethnicity of the patients (see Supplementary Tables 9-13). Overall, HLA-Cw7 was found to be greatly over-represented from the expected in the total group and in the Caucasian cohort $(\mathrm{p}<0.001$ ) with significant positive association (Table 2 and Supplemental Tables 5 and 11). HLA-DQ5 and HLA-DQ6 (Supplementary Tables 8 and 13) were nominally statistically significant but did not achieve statistical significance at the increased level we set at $\mathrm{p}<0.001$. HLA-A, HLA-B, HLA-DR, and HLA-DQ loci were not found to be represented with a significantly different frequency in ASD as compared to the general population (see Supplementary Tables 3, 4, 6, 7, 9, 10, 12). Likewise, loci on HLA-C and HLA-DQ other than those mentioned above were not found to be represented with a significantly different frequency in ASD as compared to the general population (see Supplementary Tables 5, 8, 11, 13).

The HLA types from specimens from 143 subjects with ASD were analyzed and compared with the expected distributions of HLA types in the normal population. Only HLA-Cw7 was found to be increased with respect to the expected distributions. Further, this was primarily due to more Caucasians with ASD expressing HLA-Cw7. Two hundred eighty-six alleles are present in 143 subjects. The expected allele frequency was calculated from the known distributions in the United States population and then adjusted for the frequencies of the race/ethnic frequencies in the ASD cohort. Samples with large numbers were analyzed by ${ }^{*}$ chi square, and those with small sample numbers were analyzed by ${ }^{* *}$ Fischer Exact Test. $\mathrm{P}<0.001$ was considered for statistical significance (Supplemental Tables 2-13).

In patients with ASD, HLA-C locus group II activating alleles are found more frequently than expected in the general population (Table 3 and Supplementary Table 5). This increase in HLA-C locus activating alleles is primarily due to the increased frequency of expression of HLA-Cw7 (Table 3). The increase is due to the greater expression in Caucasians (Table 4; Supplementary Table 11).

The HLA-C locus components from subjects with ASD compared with the expected frequencies indicate an increase in group II Activating HLA-Cw7. All others are not statistically significantly different from the expected frequencies.

The total numbers of group I and group II activating alleles are compared by race/ethnicity in subjects with ASD. The total

TABLE 2 | HLA-CW7 is found more than expected in patients with ASD.

\begin{tabular}{lccc}
\hline & $\begin{array}{c}\text { ASD } \\
\text { expected }\end{array}$ & $\begin{array}{c}\text { ASD } \\
\text { observed }\end{array}$ & $\mathbf{p}=$ \\
\hline Total ASD alleles $=\mathbf{2 8 6}$ & 42 & 91 & ${ }^{*} 0.000001$ \\
Caucasian alleles $=\mathbf{2 4 2}$ & 35 & 84 & ${ }^{*} 0.0000002$ \\
African American alleles = 18 & 1 & 2 & ${ }^{* *} 0.3857$ \\
Hispanic alleles = $\mathbf{8}$ & 0 & 0 & ${ }^{* *} 1.0000$ \\
Asian alleles $=\mathbf{1 8}$ & 9 & 5 & ${ }^{* *} 0.1097$ \\
\hline
\end{tabular}

${ }^{*} p$ value by Chi Square, ${ }^{* *} p$ value by Fisher Exact Test. 
TABLE 3 | Increase in patients with ASD expressing HLA-CW7 explains the increase in HLA-C group II activating alleles detected.

\begin{tabular}{|c|c|c|c|c|c|c|c|c|}
\hline HLA-C & Total expected ASD & $\begin{array}{c}\text { Group I } \\
\text { activating }\end{array}$ & Group II activating & $\begin{array}{c}\text { Total observed } \\
\text { ASD }\end{array}$ & $\begin{array}{c}\text { Group I } \\
\text { activating }\end{array}$ & $\begin{array}{c}\text { Group II } \\
\text { activating }\end{array}$ & $\mathbf{p}=$ & HLA-C \\
\hline 1 & 10 & & 10 & 10 & & 10 & ${ }^{*} 1.00$ & 1 \\
\hline 2 & 10 & 10 & & 10 & 10 & & ${ }^{*} 1.00$ & 2 \\
\hline 4 & 32 & 32 & & 32 & 32 & & **1.00 & 4 \\
\hline 5 & 24 & 24 & & 18 & 18 & & ${ }^{\star \star} 0.86$ & 5 \\
\hline 6 & 24 & 24 & & 25 & 25 & & $* * 1.00$ & 6 \\
\hline 7 & 42 & & 42 & 91 & & 91 & ${ }^{\star *} 0.0007$ & 7 \\
\hline 8 & 12 & & 12 & 11 & & 11 & $* \star 1.00$ & 8 \\
\hline 9 & 19 & & 19 & 19 & & 19 & **1.00 & 9 \\
\hline 10 & 26 & & 26 & 28 & & 28 & ${ }^{\star *} 1.00$ & 10 \\
\hline 12 & 16 & & 16 & 17 & & 17 & $* \star 1.00$ & 12 \\
\hline 14 & 7 & & 7 & 2 & & 2 & ${ }^{*} 0.44$ & 14 \\
\hline 15 & 8 & 8 & & 5 & 5 & & ${ }^{*} 0.88$ & 15 \\
\hline 16 & 9 & 9 & & 11 & 11 & & ${ }^{* \star} 0.98$ & 16 \\
\hline 17 & 5 & 5 & & 3 & 3 & & ${ }^{*} 0.92$ & 17 \\
\hline 18 & 1 & 1 & & 2 & 2 & & ${ }^{*} 0.95$ & 18 \\
\hline
\end{tabular}

*p value by Chi Square, ${ }^{* *} p$ value by Fisher Exact Test.

number of group II activating alleles is greater than expected in the total ASD cohort and the effect appears to be driven by Caucasians.

HLA-DQ5 and HLA-DQ6 were also found to trend toward being found in greater proportions in the subjects with ASD but did not achieve $\mathrm{p}<0.001$ statistical significance in this study (see Supplementary Tables 8 and 13). Inheritance of HLA-DQ6 has been previously associated with risk for scleroderma and narcolepsy. More subjects in a larger study may help to clarify the meaningfulness of this observation with regard to ASD.

\section{Clinical Characteristics of HLA Subgroups}

One hundred eight participants had information on all clinical measures in order to enter into the PLSDA. Given the significant effect of the HLA-Cw7 allele, we divided the ASD participants in those that were negative for the HLA-Cw7 allele $(\mathrm{N}=50)$ and those with at least one HLA-Cw7 allele $(\mathrm{N}=58)$. The PLSDA found two components with the first and second components explaining 9 and $12 \%$ of the variance, respectively. The discriminant function demonstrated a $65 \%$ accuracy in discriminating the groups. The individual participant's values on the two components are depicted in Supplemental Figure 1, and the correspondence of each clinical characteristic loading on the two components is given in Supplemental Figure 2.

TABLE 4 | Over-representation of HLA-C group II activating alleles observed in ASD.

\begin{tabular}{lccc}
\hline & $\begin{array}{c}\text { Total group I } \\
\text { activating alleles }\end{array}$ & $\begin{array}{c}\text { Total group II } \\
\text { activating alleles }\end{array}$ & $\mathbf{p}=$ \\
\hline Total ASD & 95 & 189 & ${ }^{*} 0.000002$ \\
Caucasian & 73 & 167 & ${ }^{*} 0.0000002$ \\
African American & 9 & 9 & ${ }^{* *} 0.26$ \\
Hispanic & 6 & 2 & ${ }^{* *} 0.06$ \\
Asian & 7 & 11 & ${ }^{* *} 0.11$ \\
\hline
\end{tabular}

${ }^{*} p$ value from Chi Square, ${ }^{* *} p$ value from Fisher Exact Test.
Supplemental Table 15 outlines the loading for the two individual components of the discriminant function as well as the total loading for both components. This is sorted by the loading value for each clinical characteristic and presented graphically in Figure 1. Positive loadings represent characteristics that are more representative of HLA-Cw7-positive participants whereas negative loading represent characteristics more representative of the HLA-Cw7-negative participants. Considering the top loadings for those who are HLACw7-positive, we find that two of the three characteristics of HLACw7-positive participants in our sample are related to immune system activation, including allergies, food intolerances, and chronic sinusitis, whereas none of the characteristics of the HLA-Cw7negative patients involve immune system activation.

To better depict the characteristics of individual participants, we created an index to describe the number of clinical symptoms attributed to HLA-Cw7-negative and HLA-Cw7-positive participants in the analysis. A value of -1 was assigned for each of the top four clinical characteristics of HLA-Cw7-negative participants (regression, chronic constipation, headaches, abdominal pain), and a value of +1 was assigned for each of the top four clinical characteristics of HLA-Cw7-positive participants (allergies, food intolerances sinusitis, ADHD). As seen in Figure 2, HLA-Cw7-positive participants demonstrated a more positive score, on average, but also were more variable in their characteristics.

\section{DISCUSSION}

The specific etiologies responsible for causing ASD remain to be fully determined. Patients with ASD, while having an overall similar neuropsychiatric phenotype resulting in the diagnosis, have quite a variability in other clinical features and laboratory studies. It is expected that the individual's genetics influenced by environmental exposures results in the disease.

Many factors have implicated the immune system involvement in ASD. Notably, inflammatory effects on the central nervous system incriminate some alteration in immune function or dysregulation of immunity (14). Identification of specific cell 


\section{Total Loading of the Two Discrimiant Components}
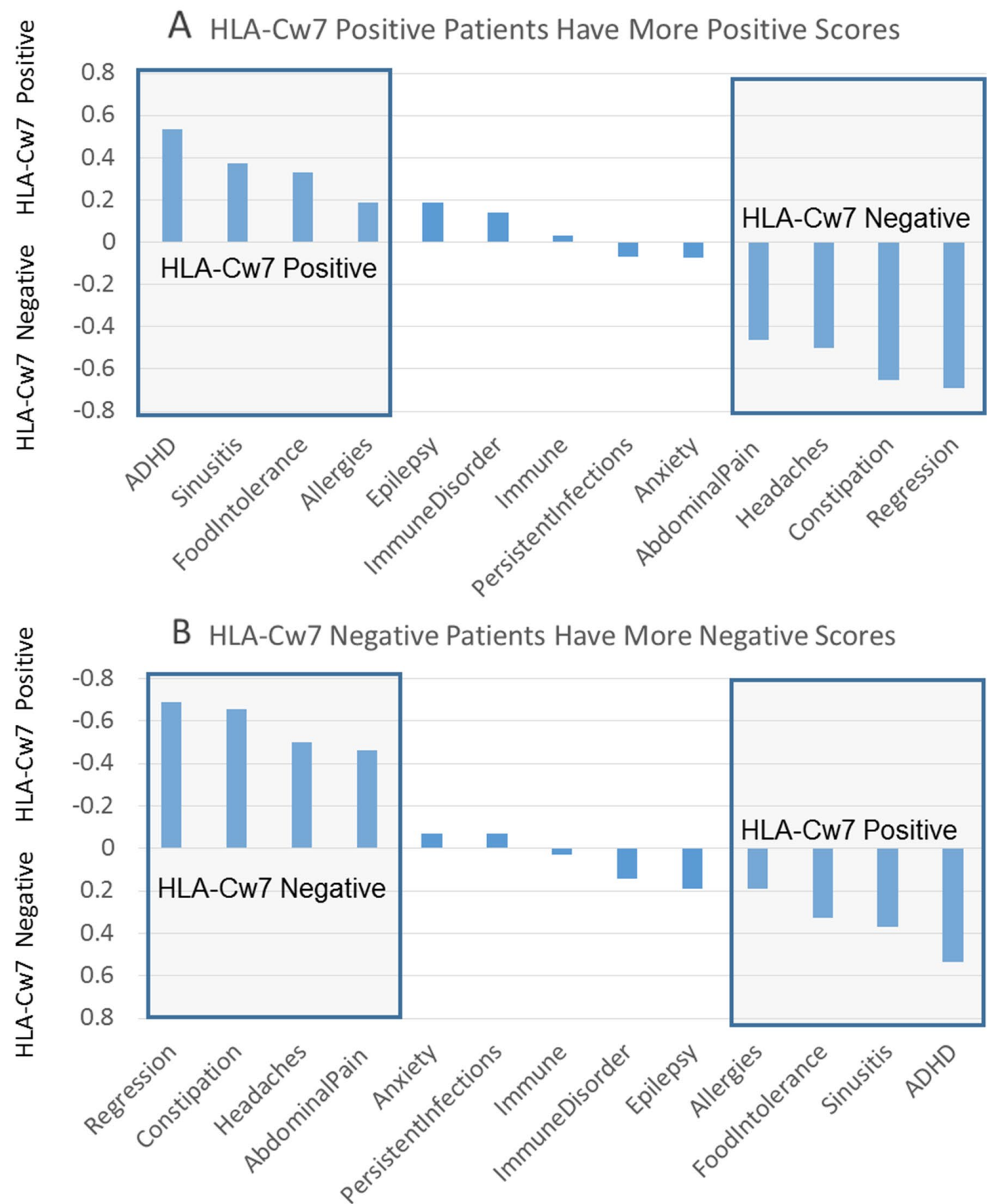

FIGURE 1 | Total components of the discriminant function. (A) Positive numbers represent components that are related to HLA-Cw7-positive participants. (B) Negative numbers represent components more representative of the HLA-CW7-negative participants.

types and targets, as can be found in specific autoimmune disorders, has not been rewarding at this point in time. Further, it is doubtful that one specific immune dysfunctional cell type and target are responsible for all ASD. It is reasonable and plausible that specific cohorts of individuals with ASD united by similar clinical and laboratory features may have similar underlying forms of immune dysfunction resulting in some form of chronic inflammation, which results in the neurologic features of disease. Since ASD tends to occur early in life, the genetic features and environmental exposures would have to be part of this early presentation. Further, since HLA components are frequently found associated with specific autoimmune disorders, are expressed on the body's cells from the onset, and shape the immune system maturation and function, it is reasonable that specific HLA components could result in the immune system's response in such an adverse manner.

Previous studies have implicated specific HLA types to be associated with ASD (6-13). Perhaps, HLA-DR4 has been most commonly found in association $(7,8,10,11)$. HLA-A2 has also been commonly reported $(6,9,10)$. Specific HLA haplotypes 


\section{Individual Characteristics}

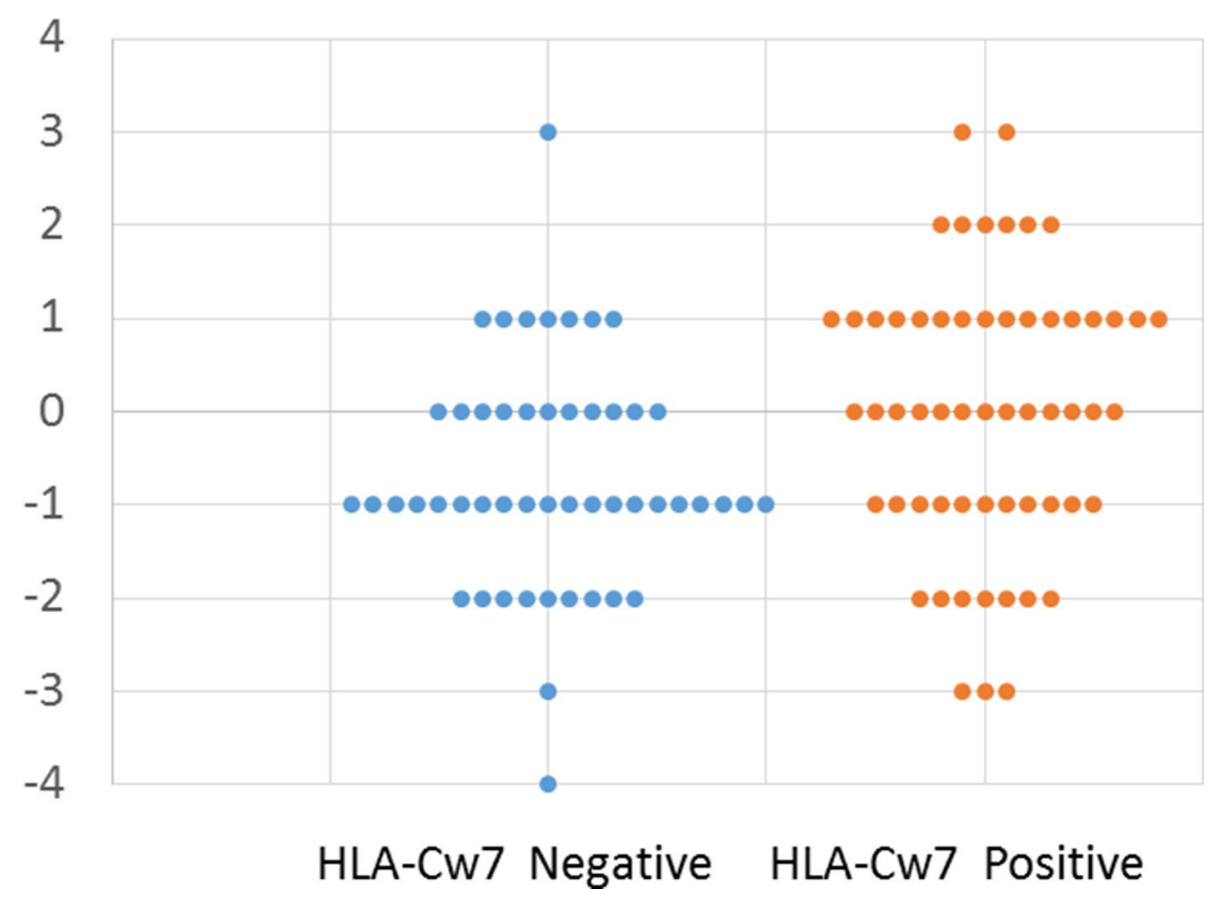

FIGURE 2 | Number of characteristics for HLA-CW7-negative and HLA-CW7-positive participants. Clinical characteristics that are more representative of absence of HLA-CW7 indicate weighted negative, and clinical characteristics of presence of HLA-CW7 indicate weighted positive.

have been reported $\left(\mathrm{HLA}-\mathrm{A}^{\star} 01-\mathrm{B}^{\star} 07-\mathrm{DRB} 1^{\star} 0701-\mathrm{DQB} 1^{\star} 0602\right)$ in patients from Saudi Arabia, and HLA-A2-B44-DR4 and HLAB44-C30-DR4, from different studies in the United States (1012). At the stringent $p$ value of $p<0.001$, we were unable to detect any of these associations (Supplemental Tables 3-13). From these reports, it may be ascertained that different geographic locations of patients with ASD may had predilection for different HLA type preferences. Although not specifically studied or stated in these reports, post-streptococcal immunoreactivity is correlated with HLA-DR4, and it is unknown whether PANDAS could be having a role in the bias toward finding an association with HLA-DR4 in some of the studies. Further, studies have not specifically sought for HLA-C locus associations, until the present study. Additionally, other studies have not specifically adjusted the expected control group HLA frequencies to the race/ ethnic frequencies found in the ASD subjects. We believe that the careful adjustment for the race/ethnic distributions of the HLA types between the control/expected HLA distributions and the observed HLA types in our subjects with ASD has resulted in the difference found in our study versus those previously reported.

We found that a cohort of patients with ASD (primarily Caucasian) expresses HLA-Cw7 at more than twice the extent expected in the normal population. These patients share immune-associated clinical features, not found in patients who do not express HLA-Cw7. Interestingly, others have associated a subset of individuals with ASD and non-IgE-mediated food allergy (40) and chronic sinusitis (41) who have dysregulation in cytokine production and microRNA expression and dysregulation of innate immunity, although HLA distribution has not been studied in this subset. While HLA-DQ5 and HLA-DQ6 trended toward greater presence in subjects with ASD, since statistical significance was not achieved, we await further analyses with a larger number of subjects with ASD to pursue further the potential meaningfulness of these findings.

The HLA-C locus is expressed on all cell types (except RBC) and has a major role in immunosurveillance. HLA-C interacts with NK cell KIR ligands in an activating and inhibiting fashion. Thus, when an NK cell interrogates a cell in the body, the correct expression of HLA-C on the body cell prevents the NK cell from becoming activated. When a cell becomes infected with a virus, one of the viral strategies to prevent recognition is to decrease the expression of HLA on the cell surface. In doing so, the alteration in HLA-C expression can alert NK cells to attack. Cancerous cells are thought to be targeted by NK cells in a similar fashion, i.e., alteration in the normal levels of HLA-C expression.

The increased frequency of subjects with HLA-Cw7 expression also results in these subjects having an overall greater expression of the HLA-C group II activating alleles. While it remains to be proven, we suspect this cohort of patients may have a continuous and ongoing level of inflammation generated by NK cells in response to this increase in group II activating alleles. This could result in the overall increase in immune-associated clinical features in this cohort. Warren et al. (17) first investigated NK cell cytotoxic activity in ASD in 1987 and found reduced ex vivo 
NK cell cytotoxic activity in $12 / 31$ ASD subjects examined. This finding was supported by Vojdani et al. (18) who demonstrated reduced ex vivo NK cell cytotoxic activity in $45 \%$ of ASD children in a much larger cohort (1,027 ASD subjects). Shortly thereafter, Enstrom et al. (19) demonstrated increased numbers of NK cells as well as upregulation of NK cell receptors and cytolytic effector molecules in 52 children with ASD as compared to 27 controls. Importantly, while ex vivo NK cell cytotoxic activity was reduced in the ASD cohort, interferon-gamma-producing NK cells were increased under resting conditions in children with ASD suggesting that NK cells in the ASD cohort are maximally activated in vivo and, thus, chronically activated NK cells are down-regulated when stimulated ex vivo. Unfortunately, HLA typing was not performed in these three studies. Taken together, these data support the notion that a subset of ASD subjects with greater expression of HLA-C group II activating alleles may have chronically activated NK cells. Torres et al. have previously reported the association of activating KIR ligands and ASD (11, 13). Indeed, the KIR-activating gene 2DS1 was shown to be increased in frequency in patients with ASD, and this supports our findings as HLA-Cw7 is a cognate ligand for KIR 2DS1 (13). Thus, this could result in a greater level of NK cell activation in patients with ASD who express HLA-Cw7.

Further studies in patients with ASD who express HLA-Cw7 are needed with specific evaluation of NK cell activation in vivo. This could potentially lead to therapeutic intervention by specifically targeting over-active NK cells in patients with ASD who express HLA-Cw7. The overall decrease in chronic inflammation could then decrease the burden on the central nervous system in a cohort of patients with ASD.

\section{ETHICS STATEMENT}

The Institutional Review Board (IRB) at the University of Arkansas for Medical Sciences (Little Rock, AR) approved the clinical studies and use of cell lines. For clinical studies, parents of participants provided written informed consent. All experiments were performed in accordance with relevant guidelines and regulations.

\section{REFERENCES}

1. Christensen DL, Braun KVN, Baio J, Bilder D, Charles J, Constantino JN, et al. Prevalence and characteristics of autism spectrum disorder among children aged 8 years-autism and developmental disabilities monitoring network, 11 sites, United States, 2012. MMWR Surveill Summ (2018) 65(13):1-23. doi: 10.15585/mmwr.ss6513al

2. Wiggins L, Christensen D, Van Naarden Braun K, Martin L, Baio J. Comparison of autism spectrum disorder surveillance status based on two different diagnostic schemes: findings from the metropolitan atlanta developmental disabilities surveillance program, 2012. PLoS One (2018) 13(11):e0208079. doi: 10.1371/journal.pone.0208079

3. Rossignol DA, Frye RE. A review of research trends in physiological abnormalities in autism spectrum disorders: immune dysregulation, inflammation, oxidative stress, mitochondrial dysfunction and environmental toxicant exposures. $\mathrm{Mol}$ Psychiatry (2012) 17(4):389-401. doi: 10.1038/mp.2011.165

4. Rossignol DA, Genuis SJ, Frye RE. Environmental toxicants and autism spectrum disorders: a systematic review. Transl Psychiatry (2014) 4:e360. doi: $10.1038 /$ tp.2014.4

\section{AUTHOR CONTRIBUTIONS}

All authors were involved in the design and conceptualization of the experiments. Laboratory experiments were conducted by TH, BR-C, SB, RW and SR. LD, JS, MT and RF were involved in subject recruitment and assessment. Data was analyzed by $\mathrm{TH}, \mathrm{LD}, \mathrm{SR}, \mathrm{SK}$ and RF. All authors were involved in drafting, editing and finalizing the manuscript. All authors approved of the final manuscript.

\section{FUNDING}

We would like to thank the supporters of our research studies, including the Arkansas Biosciences Institute (Little Rock, AR, USA), the Jonty Foundation (St. Paul, MN), the Autism Research Institute (San Diego, CA), the Gupta Family Foundation (Atherton, CA), the Jane Bostford Johnson Foundation (New York, NY), the Jager Family Foundation (Chicago, IL) and the Phoenix Children's Hospital Foundation (Phoenix, AZ). HLA typing was in part funded by Department of Pathology, UAMS, Research Funds.

\section{ACKNOWLEDGMENTS}

We thank the families and individuals with ASD that participated in the Autism Genetic Research Exchange and the studies at the National Institutes of Mental Health for providing cell lines for research as well as families and individuals that were involved in clinical research at the Arkansas Children's Research Institute (Little Rock, AR).

\section{SUPPLEMENTARY MATERIAL}

The Supplementary Material for this article can be found online at: https://www.frontiersin.org/articles/10.3389/fpsyt.2019.00612/ full\#supplementary-material

5. McDougle CJ, Landino SM, Vahabzadeh A, O'Rourke J, Zurcher NR, Finger BC, et al. Toward an immune-mediated subtype of autism spectrum disorder. Brain Res (2015) 1617:72-92. doi: 10.1016/j.brainres.2014.09.048

6. Stubbs EG, Magenis RE. HLA and autism. J Autism Dev Disord (1980) 10(1):15-9.

7. Torres AR, Maciulis A, Stubbs EG, Cutler A, Odell D. The transmission disequilibrium test suggests that HLA-DR4 and DR13 are linked to autism spectrum disorder. Hum Immunol (2002) 63(4):311-6.

8. Lee LC, Zachary AA, Leffell MS, Newschaffer CJ, Matteson KJ, Tyler JD, et al. HLA-DR4 in families with autism. Pediatr Neurol (2006) 35(5):303-7. doi: 10.1016/j.pediatrneurol.2006.06.006

9. Torres AR, Sweeten TL, Cutler A, Bedke BJ, Fillmore M, Stubbs EG, et al. The association and linkage of the HLA-A2 class I allele with autism. Hum Immunol (2006) 67(4-5):346-51. doi: 10.1016/j.humimm.2006.01.001

10. Chien YL, Wu YY, Chen CH, Gau SS, Huang YS, Chien WH, et al. Association of HLA-DRB1 alleles and neuropsychological function in autism. Psychiatr Genet (2012) 22(1):46-9. doi: 10.1097/YPG.0b013e32834915ae

11. Torres AR, Westover JB, Gibbons C, Johnson RC, Ward DC. Activating killer-cell immunoglobulin-like receptors (KIR) and their cognate HLA 
ligands are significantly increased in autism. Brain Behav Immun (2012) 26(7):1122-7. doi: 10.1016/j.bbi.2012.07.014

12. Al-Hakbany M, Awadallah S, Al-Ayadhi L. The relationship of HLA class I and II alleles and haplotypes with autism: a case control study. Autism Res Treat (2014) 2014:242048. doi: 10.1155/2014/242048

13. Torres AR, Sweeten TL, Johnson RC, Odell D, Westover JB, Bray-Ward P, et al. Common genetic variants found in HLA and KIR immune genes in autism spectrum disorder. Front Neurosci (2016) 10:463. doi: 10.3389/ fnins. 2016.00463

14. Edmiston E, Ashwood P, Van de Water J. Autoimmunity, autoantibodies, and autism spectrum disorder. Biol Psychiatry (2017) 81(5):383-90. doi: 10.1016/j.biopsych.2016.08.031

15. Connery K, Tippett M, Delhey LM, Rose S, Slattery JC, Kahler SG, et al. Intravenous immunoglobulin for the treatment of autoimmune encephalopathy in children with autism. Transl Psychiatry (2018) 8(1):148. doi: 10.1038/s41398-018-0214-7

16. Vargas DL, Nascimbene C, Krishnan C, Zimmerman AW, Pardo CA. Neuroglial activation and neuroinflammation in the brain of patients with autism. Ann Neurol (2005) 57(1):67-81. doi: 10.1002/ana.20315

17. Warren RP, Foster A, Margaretten NC. Reduced natural killer cell activity in autism. J Am Acad Child Adolesc Psychiatry (1987) 26(3):333-5. doi: 10.1097/00004583-198705000-00008

18. Vojdani A, Mumper E, Granpeesheh D, Mielke L, Traver D, Bock K, et al. Low natural killer cell cytotoxic activity in autism: the role of glutathione, IL-2 and IL-15. J Neuroimmunol (2008) 205(1-2):148-54. doi: 10.1016/ j.jneuroim.2008.09.005

19. Enstrom AM, Lit L, Onore CE, Gregg JP, Hansen RL, Pessah IN, et al. Altered gene expression and function of peripheral blood natural killer cells in children with autism. Brain Behav Immun (2009) 23(1):124-33. doi: 10.1016/j.bbi.2008.08.001

20. Frye RE, Delhey L, Slattery J, Tippett M, Wynne R, Rose S, et al. Blocking and binding folate receptor alpha autoantibodies identify novel autism spectrum disorder subgroups. Front Neurosci (2016) 10:80. doi: 10.3389/ fnins. 2016.00080

21. Frye RE, Slattery J, Delhey L, Furgerson B, Strickland T, Tippett M, et al. Folinic acid improves verbal communication in children with autism and language impairment: a randomized double-blind placebo-controlled trial. Mol Psychiatry (2018) 23(2):247-56. doi: 10.1038/mp.2016.168

22. Burger BJ, Rose S, Bennuri SC, Gill PS, Tippett ML, Delhey L, et al. Autistic siblings with novel mutations in two different genes: insight for genetic workups of autistic siblings and connection to mitochondrial dysfunction. Front Pediatr (2017) 5:219. doi: 10.3389/fped.2017.00219

23. Delhey L, Kilinc EN, Yin L, Slattery J, Tippett M, Wynne R, et al. Bioenergetic variation is related to autism symptomatology. Metab Brain Dis (2017) 32(6):2021-31. doi: 10.1007/s11011-017-0087-0

24. Delhey LM, Nur Kilinc E, Yin L, Slattery JC, Tippett ML, Rose S, et al. The effect of mitochondrial supplements on mitochondrial activity in children with autism spectrum disorder. J Clin Med (2017) 6(2):pii:E18. doi: 10.3390/ jcm6020018

25. Frye RE, Wynne R, Rose S, Slattery J, Delhey L, Tippett M, et al. Thyroid dysfunction in children with autism spectrum disorder is associated with folate receptor alpha autoimmune disorder. J Neuroendocrinol (2017) 29(3) :1-12. doi: 10.1111/jne.12461

26. Delhey LM, Tippett M, Rose S, Bennuri SC, Slattery JC, Melnyk S, et al. Comparison of treatment for metabolic disorders associated with autism:reanalysis of three clinical trials. Front Neurosci (2018) 12:19. doi: 10.3389/fnins.2018.00019

27. Howsmon DP, Vargason T, Rubin RA, Delhey L, Tippett M, Rose S, et al. Multivariate techniques enable a biochemical classification of children with autism spectrum disorder versus typically-developing peers: a comparison and validation study. Bioeng Transl Med (2018) 3(2):156-65. doi: 10.1002/ btm2.10095

28. Vargason T, Kruger U, Roth E, Delhey LM, Tippett M, Rose S, et al. Comparison of three clinical trial treatments for autism spectrum disorder through multivariate analysis of changes in metabolic profiles and adaptive behavior. Front Cell Neurosci (2018) 12:503. doi: 10.3389/fncel.2018.00503

29. Rose S, Frye RE, Slattery J, Wynne R, Tippett M, Melnyk S, et al. Oxidative stress induces mitochondrial dysfunction in a subset of autistic lymphoblastoid cell lines. Transl Psychiatry (2014) 4:e377. doi: 10.1038/ tp.2014.15

30. Rose S, Frye RE, Slattery J, Wynne R, Tippett M, Pavliv O, et al. Oxidative stress induces mitochondrial dysfunction in a subset of autism lymphoblastoid cell lines in a well-matched case control cohort. PLoS One (2014) 9(1):e85436. doi: 10.1371/journal.pone.0085436

31. Rose S, Bennuri SC, Wynne R, Melnyk S, James SJ, Frye RE. Mitochondrial and redox abnormalities in autism lymphoblastoid cells: a sibling control study. FASEB J (2017) 31(3):904-9. doi: 10.1096/fj.201601004R

32. Bennuri SC, Rose S, Frye RE. Mitochondrial dysfunction is inducible in lymphoblastoid cell lines from children with autism and may involve the TORC1 pathway. Front Psychiatry (2019) 10:269. doi: 10.3389/fpsyt.2019.00269

33. Rose S, Wynne R, Frye RE, Melnyk S, James SJ. Increased susceptibility to ethylmercury-induced mitochondrial dysfunction in a subset of autism lymphoblastoid cell lines. J Toxicol (2015) 2015:573701. doi: 10.1155/2015/573701

34. Frye RE, Rose S, Chacko J, Wynne R, Bennuri SC, Slattery JC, et al. Modulation of mitochondrial function by the microbiome metabolite propionic acid in autism and control cell lines. Transl Psychiatry (2016) 6(10):e927. doi: 10.1038/tp.2016.189

35. Frye RE, Rose S, Wynne R, Bennuri SC, Blossom S, Gilbert KM, et al. Oxidative stress challenge uncovers trichloroacetaldehyde hydrate-induced mitoplasticity in autistic and control lymphoblastoid cell lines. Sci Rep (2017) 7(1):4478. doi: 10.1038/s41598-017-04821-3

36. Rose S, Bennuri SC, Davis JE, Wynne R, Slattery JC, Tippett M, et al. Butyrate enhances mitochondrial function during oxidative stress in cell lines from boys with autism. Transl Psychiatry (2018) 8(1):42. doi: 10.1038/ s41398-017-0089-z

37. Frye RE, Nankova B, Bhattacharyya S, Rose S, Bennuri SC, MacFabe DF. Modulation of immunological pathways in autistic and neurotypical lymphoblastoid cell lines by the enteric microbiome metabolite propionic acid. Front Immunol (2017) 8:1670. doi: 10.3389/fimmu.2017.01670

38. Harville TO. "HLA typing for cellular product characterization and identity testing," In: EllenAreman MM, Loper K, editors. Cellular Therapy: Principles, Methods, and Regulations. AABB (2016).

39. Batra A, Cottler-Fox M, Harville T, Rhodes-Clark BS, Makhoul I, Nakagawa M. Autologous graft versus host disease: an emerging complication in patients with multiple myeloma. Bone Marrow Res (2014) 2014:891427. doi: 10.1155/ 2014/891427

40. Jyonouchi H, Geng L, Streck DL, Dermody JJ, Toruner GA. MicroRNA expression changes in association with changes in interleukin-1ss/interleukin 10 ratios produced by monocytes in autism spectrum disorders: their association with neuropsychiatric symptoms and comorbid conditions (observational study). J Neuroinflammation (2017) 14(1):229. doi: 10.1186/s12974-017-1003-6

41. Jyonouchi H, Geng L, Streck DL, Toruner GA. Immunological characterization and transcription profiling of peripheral blood (PB) monocytes in children with autism spectrum disorders (ASD) and specific polysaccharide antibody deficiency (SPAD): case study. J Neuroinflammation (2012) 9:4. doi: 10.1186/1742-2094-9-4

Conflict of Interest Statement: JS is the Co-Founder, President and Chief Executive Officer of BioRosa Technologies Inc, a start-up company focused on developing a diagnostic test for ASD based on metabolic biomarkers. JS contributed in the collection of participant data during his employment at the University of Arkansas for Medical Sciences as the clinical trials manager for the autism research program at Arkansas Children's Research Institute. No data or samples were shared with JS or BioRosa for commerical use.

The remaining authors declare that the research was conducted in the absence of any commercial or financial relationships that could be construed as a potential conflict of interest.

Copyright (c) 2019 Harville, Rhodes-Clark, Bennuri, Delhey, Slattery, Tippett, Wynne, Rose, Kahler and Frye. This is an open-access article distributed under the terms of the Creative Commons Attribution License (CC BY). The use, distribution or reproduction in other forums is permitted, provided the original author(s) and the copyright owner(s) are credited and that the original publication in this journal is cited, in accordance with accepted academic practice. No use, distribution or reproduction is permitted which does not comply with these terms. 